\title{
Assessment of subacromial space and its relationship with scapular upward rotation in college baseball players.
}

\author{
Stephen John Thomas \\ University of Pennsylvania \\ Charles Buz Swanik \\ Dept of Health, Nutrition and Exercise Science, University of Delaware \\ Thomas W Kaminski \\ Dept of Health, Nutrition and Exercise Science, University of Delaware \\ Jill S Higginson \\ Dept of Mechanical Engineering, University of Delaware
}

Katbleem AA Swandiktional works at: https://jdc.jefferson.edu/radiologyfp neot of Athletic Training, Neumann University

Part of the Orthopedics Commons

\section{Let us know how access to this document benefits you}

\section{Recommended Citation}

Thomas, Stephen John; Swanik, Charles Buz; Kaminski, Thomas W; Higginson, Jill S; Swanik, Kathleen A; and Nazarian, Levon N, "Assessment of subacromial space and its relationship with scapular upward rotation in college baseball players." (2013). Department of Radiology Faculty Papers. Paper 33.

https://jdc.jefferson.edu/radiologyfp/33

This Article is brought to you for free and open access by the Jefferson Digital Commons. The Jefferson Digital Commons is a service of Thomas Jefferson University's Center for Teaching and Learning (CTL). The Commons is a showcase for Jefferson books and journals, peer-reviewed scholarly publications, unique historical collections from the University archives, and teaching tools. The Jefferson Digital Commons allows researchers and interested readers anywhere in the world to learn about and keep up to date with Jefferson scholarship. This article has been accepted for inclusion in Department of Radiology Faculty Papers by an authorized administrator of the Jefferson Digital Commons. For more information, please contact: JeffersonDigitalCommons@jefferson.edu. 


\section{Authors}

Stephen John Thomas, Charles Buz Swanik, Thomas W Kaminski, Jill S Higginson, Kathleen A Swanik, and Levon N Nazarian 


\title{
Assessment of Subacromial Space and Its Relationship With Scapular Upward Rotation in College Baseball Players
}

\author{
Stephen John Thomas, Charles Buz Swanik, Thomas W. Kaminski, Jill S. Higginson, \\ Kathleen A. Swanik, and Levon N. Nazarian
}

\begin{abstract}
Context: Subacromial impingement is a common injury in baseball players and has been linked to a reduction in the subacromial space. In addition, it has been suggested that decreases in scapular upward rotation will lead to decreases in the subacromial space and ultimately impingement syndrome. Objective: The objective of this study was to evaluate the relationship between acromiohumeral distance and scapular upward rotation in healthy college baseball players. Design: Posttest-only study design. Setting: Controlled laboratory setting. Participants: 24 healthy college baseball players. Intervention: Participants were measured for all dependent variables at preseason. Main Outcome Measures: Acromiohumeral distance at rest and $90^{\circ}$ of abduction was measured with a diagnostic ultrasound unit. Scapular upward rotation at rest and $90^{\circ}$ of abduction was measured with a digital inclinometer. Results: Dominant-arm acromiohumeral distance at rest and $90^{\circ}$ of abduction $(P=.694, P=.840)$ was not significantly different than in the nondominant arm. In addition, there was not a significant correlation between acromiohumeral distance and scapular upward rotation at rest and $90^{\circ}$ of abduction for either the dominant or the nondominant arm. Conclusions: These results indicate that the acromiohumeral distance is not adapting in the dominant arm in healthy throwing athletes. In addition, a relationship was not identified between acromiohumeral distance and scapular upward rotation, which was previously suggested. These results may suggest that changes that are typically seen in an injured population may be occurring due to the injury and are not preexisting. In addition, scapular upward rotation may not be the only contributing factor to acromiohumeral distance.
\end{abstract}

Keywords: shoulder, ultrasound, rotator cuff, range of motion

Clinically, subacromial impingement syndrome is a common injury among overhead athletes. ${ }^{1-6}$ It is typically defined as an entrapment of the soft-tissue structures in the subacromial space during arm elevation. ${ }^{7}$ Any decrease of the subacromial space may lead to a compression of the soft-tissue structures, thereby leading to impingement pain. In the past, impingement syndrome has been suggested to be associated with anterior instability in baseball players. ${ }^{4-6}$ However, recent data have demonstrated altered scapular kinematics in subjects with impingement syndrome, suggesting that these abnormalities may also be related to the injury. 8,9

Thomas is with Orthopaedic Surgery, University of Pennsylvania, Philadelphia, PA. C. Swanik and Kaminski are with the Dept of Health, Nutrition and Exercise Science, and Higginson, the Dept of Mechanical Engineering, University of Delaware, Newark, DE. K. Swanik is with the Dept of Athletic Training, Neumann University, Aston, PA. Nazarian is with the Dept of Radiology, Thomas Jefferson University Hospital, Philadelphia, PA.
The commonly reported scapular alterations in injured baseball players are decreased upward rotation, increased anterior tilting, protraction, and internal rotation. ${ }^{1,8,10,11}$ In previous work we have observed a decrease in scapular upward rotation over the course of a baseball season in both high school and college baseball players. ${ }^{12,13}$ We also found significantly less upward rotation and increased protraction in college than in high school baseball players. ${ }^{14}$ This suggests that the commonly seen alterations in scapular kinematics in patients with impingement are also occurring in healthy baseball players and require additional scrutiny. Biomechanically these scapular alterations have been thought to decrease the subacromial space, thereby leading to mechanical compression of the supraspinatus and subacromial bursa. ${ }^{15,16}$ This compression over time may very well cause irritation, pain, and inflammation in the subacromial space, further exacerbating the symptoms ${ }^{17}$; however, only 1 study has attempted to measure the acromiohumeral distance in college baseball players compared with control subjects. ${ }^{18}$ They found that the college baseball players had a significantly increased acromiohumeral distance at $0^{\circ}$ and $90^{\circ}$ of glenohumeral abduction, suggestive of 
a beneficial adaptive change to allow for more clearance of the subacromial structures compared with controls. ${ }^{18}$ However, that study neglected to compare the baseball players' throwing arms with their nonthrowing arms. This comparison between the throwing and nonthrowing shoulders would be informative with respect to adaptive changes or congenital differences. Furthermore, no studies have assessed acromiohumeral distance in the same subjects' dominant and nondominant arms and also correlated the acromiohumeral distance measurement with scapular upward rotation to establish the inferred mechanism for potential impingement. We hypothesized that the dominant arm would have a decreased acromiohumeral distance at both rest and $90^{\circ}$ of abduction compared with the nondominant arm. In addition, we hypothesized that there would be a positive relationship between acromiohumeral distance and scapular upward rotation at both rest and $90^{\circ}$ of abduction.

\section{Methods}

\section{Design}

A posttest-only design was used to assess 2 dependent variables and 1 independent variable. The independent variable was arm (dominant and nondominant). The dependent variables were acromiohumeral distance and scapular upward rotation at rest and at $90^{\circ}$ of glenohumeral abduction. The average of 3 measurements was taken for analysis.

\section{Participants}

Twenty-four healthy college baseball players (pitchers, $\mathrm{n}=12$, age $=19.4 \pm 1.16 \mathrm{y}$, mass $=88.14 \pm 4.81 \mathrm{~kg}$, and height $=188.38 \pm 5.61 \mathrm{~cm}$; position players, $\mathrm{n}=12$, age $=19.8 \pm 1.48 \mathrm{y}$, mass $=90.19 \pm 6.67 \mathrm{~kg}$, and height $=184.15 \pm 2.97 \mathrm{~cm}$ ) volunteered to participate in this study. Inclusionary criteria consisted of participation on an intercollegiate baseball team, age 18 to 30 years, and being healthy for the past 6 months. Exclusion criteria consisted of injury or surgery in the past 6 months, and subjects were not allowed to throw in the 5 days before testing. The study was approved by a university institutional review board. Informed consent and a healthhistory questionnaire were obtained from participants before testing.

\section{Instrumentation}

Ultrasound scanning of the acromiohumeral distance was performed with a $10-\mathrm{MHz}$ linear transducer and a commercially available compact ultrasound system (Sonosite Titan, Sonosite Inc, Bothell, WA) that has a measurement accuracy of $0.15 \mathrm{~mm}$. A priori intratester reliability of the acromiohumeral distance at $0^{\circ}$ and $90^{\circ}$ of glenohumeral abduction was assessed in a previous study by Wang et al. ${ }^{18}$ The calculated $\mathrm{ICC}_{2,1}$ values for acromiohumeral distance at $0^{\circ}$ and $90^{\circ}$ of glenohumeral abduction were .81 and .75 , respectively.
Scapular upward rotation was measured using a Saunders digital inclinometer (The Saunders Group Inc, Chaska, MN) modified to rest evenly on the scapular spine. ${ }^{19}$ The digital inclinometer was modified using methods described by Johnson et al. ${ }^{20}$ A priori test-retest reliability of the scapular upward rotation measurements was assessed by the primary investigator. Both shoulders of 18 healthy volunteers were measured and then remeasured 3 to 5 days later. The intraclass correlation coefficients (ICCs) and standard errors of the mean (SEM) for scapular upward rotation were $967\left(0.70^{\circ}\right)$ and $.974\left(0.86^{\circ}\right)$ at rest and $90^{\circ}$ of glenohumeral abduction, respectively.

\section{Procedures}

The acromiohumeral distance was measured with the subject seated upright on a stool. Care was taken to position the subject in a normal upright posture. The subject's arm was actively placed in the scapular plane $\left(30^{\circ}\right.$ anterior to the frontal plane) with 0 and $90^{\circ}$ of shoulder abduction (measured with a digital inclinometer). The ultrasound transducer was placed on the midpoint of the lateral edge of the acromion (Figures 1 and 2). The midpoint was determined by palpating the anterior and posterior aspects of the acromion. Within the ultrasound window the acromion, humeral head, and rotator cuff were identified. The acromiohumeral distance at $0^{\circ}$ of abduction was recorded as a perpendicular line from the lateralmost tip of the acromion to the humeral head (Figure 3 ). For $90^{\circ}$ of abduction the participant was instructed to actively position his shoulder in $90^{\circ}$ of abduction and $90^{\circ}$ of external rotation. This position was measured with a digital inclinometer, and participants were asked to actively hold that position during testing. Similar to the acromiohumeral distance at rest, the distance between the most lateral edge of the acromion and the humeral head was recorded for the $90^{\circ}$ position. ${ }^{18}$ When the acromiohumeral distance was identified, the image was frozen and saved to the hard drive and removed after testing was complete for analysis. The image was then opened using Image J software (Bethesda, MD), and the acromiohumeral distance was measured and recorded. This measurement was taken bilaterally for all subjects.

Scapular upward-rotation measurements were taken with the subject standing with normal relaxed posture. A guide pole was used to help position the subject's arm at $90^{\circ}$ of abduction in the scapular plane. When the appropriate amount of abduction was determined, a pin was inserted into the guide pole and the subject was instructed to abduct his arm against the pin to maintain accurate shoulder positioning. This position was maintained until the measurement was recorded. The lateral arm of the inclinometer was then placed over the posterolateral acromion and the medial arm was placed over the root of the scapular spine. The hold button was pressed to record the measurement. This was repeated twice, and the average of the 2 measurements was recorded. All measurements were taken bilaterally by the primary 

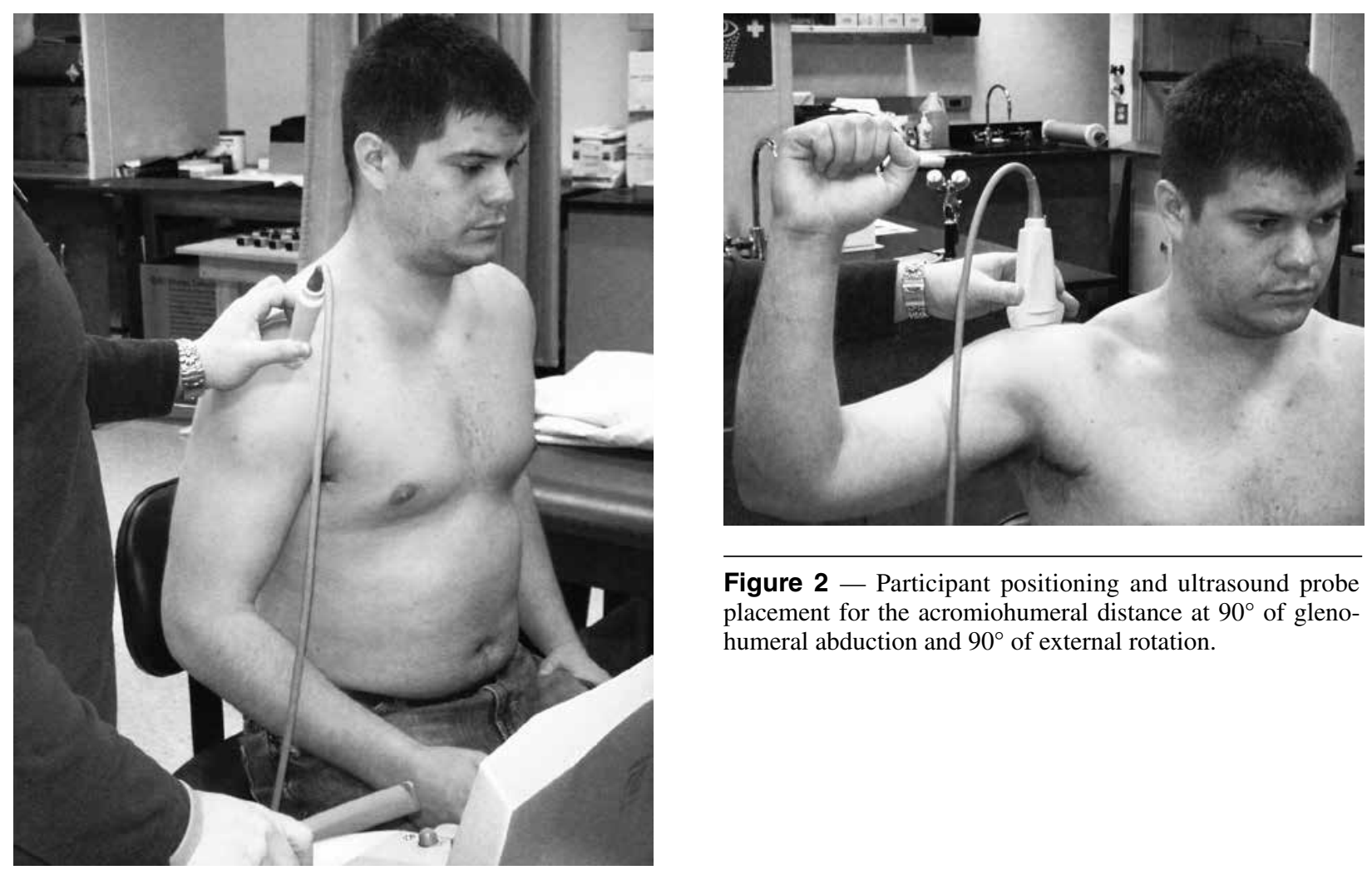

Figure 2 - Participant positioning and ultrasound probe placement for the acromiohumeral distance at $90^{\circ}$ of glenohumeral abduction and $90^{\circ}$ of external rotation.

Figure 1 - Participant positioning and ultrasound probe placement for the acromiohumeral distance at rest.

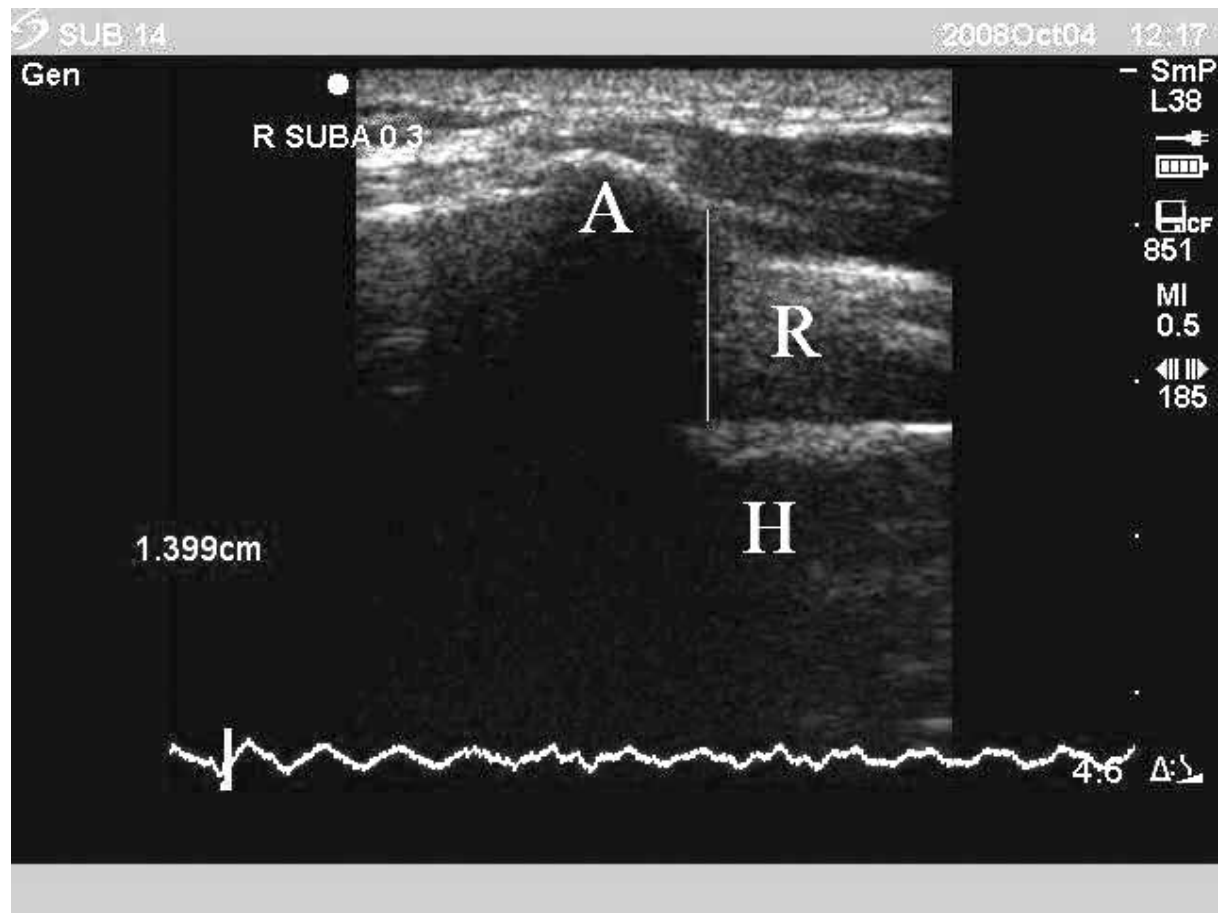

Figure 3 - Measurement of the acromiohumeral distance using ultrasound. A, acromion; R, rotator cuff; H, humeral head. The vertical line indicates the acromiohumeral distance. 
investigator, and the subjects did not perform a warm-up before the measurements. The primary investigator was blinded to the arm dominance of the athlete, and the order of testing was alternated.

\section{Statistical Analysis}

Data analysis consisted of descriptive and interferential statistics. Paired-sample $t$ tests were performed comparing dominant and nondominant arms for acromiohumeral distance. Pearson correlations were calculated relating acromiohumeral distance and scapular upward rotation. Since there were no side-to-side differences, both shoulders were pooled together for the correlation analysis to increase power.

\section{Results}

Means and standard deviations for acromiohumeral distance are presented in (Table 1). Paired-sample $t$ tests did not reveal a significant difference between the dominant arm and nondominant arm for acromiohumeral distance at $0^{\circ}$ and $90^{\circ}$ of glenohumeral abduction $(P=.7, P=$ .8). Means and standard deviations for scapular upward rotation are presented in (Table 1). Pearson correlation demonstrated a nonsignificant correlation between acromiohumeral distance at $0^{\circ}$ and scapular upward rotation at rest for both the dominant and the nondominant arm ( $r=-.02, P=.9 ; r=.35, P=.1$, respectively; Figures 4 and 5). A nonsignificant correlation was also found between acromiohumeral distance at $90^{\circ}$ and scapular upward rotation $90^{\circ}$ of glenohumeral abduction for both the dominant and the nondominant $\operatorname{arm}(r=-.09, P=.7$; $r=.1, P=.6$, respectively; Figures 6 and 7).

\section{Discussion}

The results of our study indicated that healthy college baseball players did not present with bilateral differences in acromiohumeral distance. It was surprising that correlations between acromiohumeral distance and scapular upward rotation were not found. Our findings

Table 1 Dominant- and Nondominant-Arm Subacromial Space and Scapular Upward Rotation at Rest and $90^{\circ}$ of Glenohumeral Abduction, Mean \pm SD

\begin{tabular}{lccc}
\hline Measurement & Position & Dominant arm & Nondominant arm \\
\hline Subacromial space, $\mathrm{mm}$ & $0^{\circ}$ & $12.07 \pm 1.95$ & $11.96 \pm 1.85$ \\
& $90^{\circ}$ & $12.85 \pm 2.35$ & $12.77 \pm 2.35$ \\
Scapular upward rotation, ${ }^{\circ}$ & $0^{\circ}$ & $6.23 \pm 3.26$ & $7.48 \pm 3.59$ \\
& $90^{\circ}$ & $28.11 \pm 7.22$ & $24.43 \pm 5.65$ \\
\hline
\end{tabular}

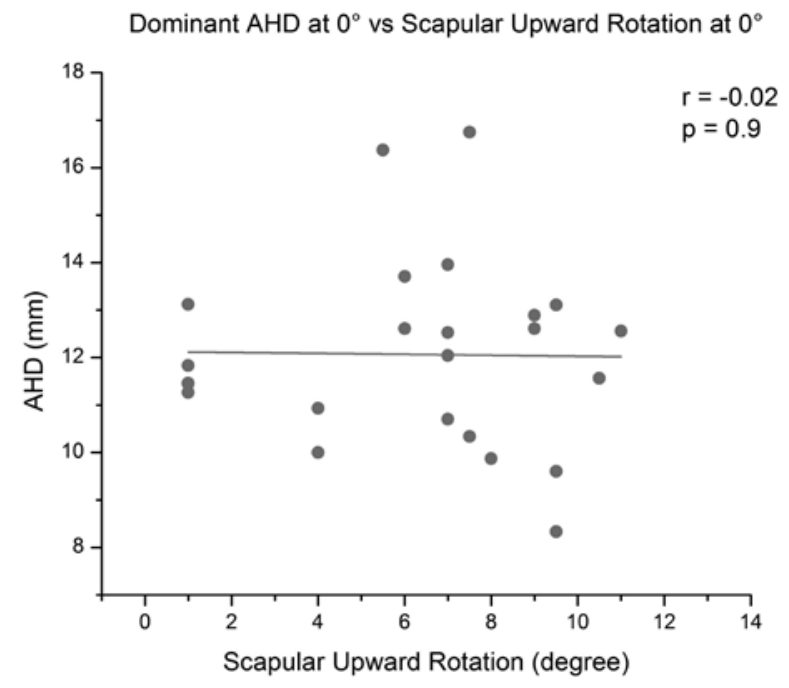

Figure 4 - Correlation between dominant-arm acromiohumeral distance (AHD) at $0^{\circ}$ and scapular upward rotation at $0^{\circ}$ of glenohumeral abduction. There was not a significant correlation.

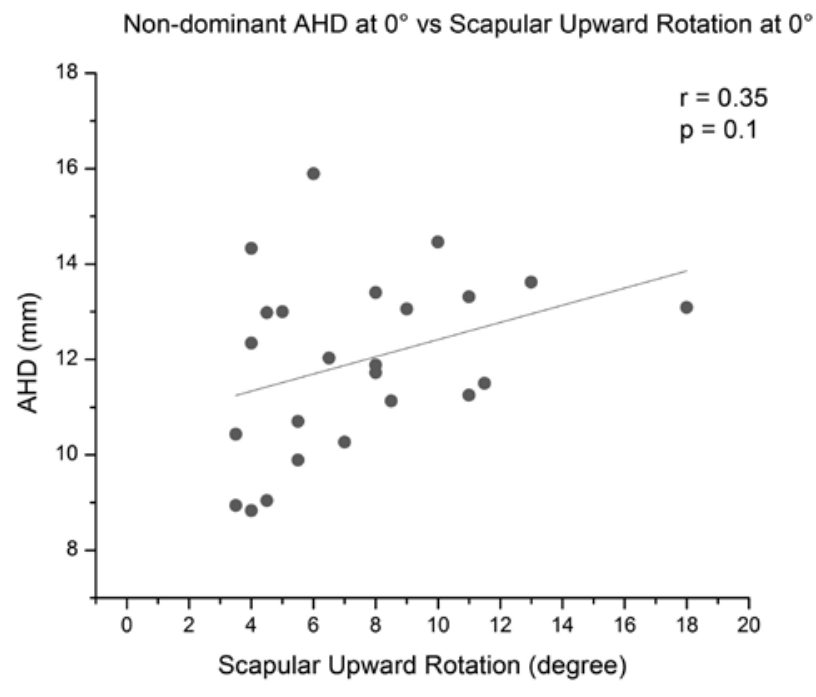

Figure 5 - Correlation between nondominant-arm acromiohumeral distance (AHD) at $0^{\circ}$ and scapular upward rotation at $0^{\circ}$ of glenohumeral abduction. There was not a significant correlation. 
Dominant $\mathrm{AHD}$ at $90^{\circ}$ vs Scapular Upward Rotation at $90^{\circ}$

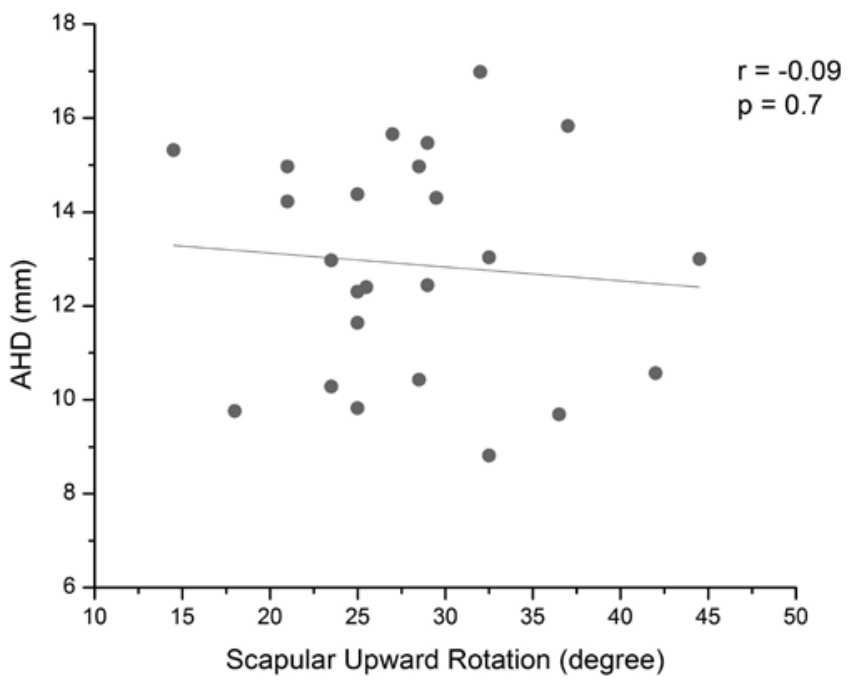

Figure 6 - Correlation between dominant-arm acromiohumeral distance (AHD) at $90^{\circ}$ and scapular upward rotation at $90^{\circ}$ of glenohumeral abduction. There was not a significant correlation.

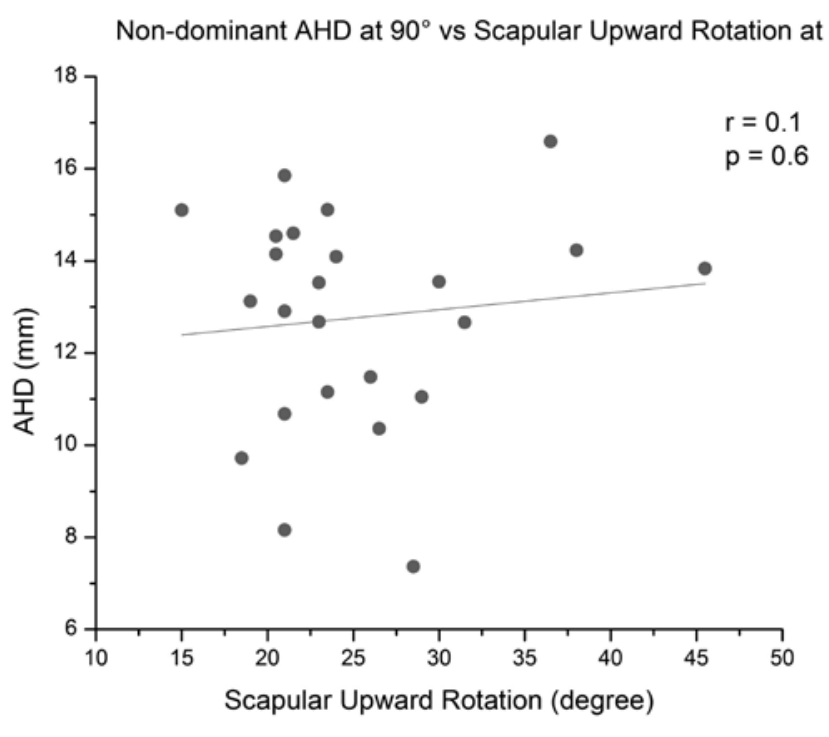

Figure 7 - Correlation between nondominant-arm acromiohumeral distance (AHD) at $90^{\circ}$ and scapular upward rotation at $90^{\circ}$ of glenohumeral abduction. There was not a significant correlation.

injuries. However, decreased acromiohumeral distance was not demonstrated at either $0^{\circ}$ or $90^{\circ}$ of glenohumeral abduction, which suggests there is no deleterious adaptation to subacromial space occurring in healthy baseball players. It may be that this healthy cohort has sufficient clearance of the rotator cuff through the subacromial arch, which would allow for minimal compression of the softtissue structures during throwing. Similar to the current study, Wang et al ${ }^{18}$ examined acromiohumeral distance in a group of college baseball players but compared them with healthy nonthrowing controls. They found that the college baseball players had significantly more acromiohumeral distance at both $0^{\circ}$ and $90^{\circ}$ of glenohumeral abduction than did the nonthrowing control group. They suggested that baseball players may adaptively increase the acromiohumeral distance through enhanced neuromuscular control of the scapula to prevent injuries due to the repetitive overhead nature of throwing. However, we also did not observe any increase in dominant-arm acromiohumeral distance, which would have been expected based on the results of Wang et al. ${ }^{18}$ However, Wang et $\mathrm{al}^{18}$ pooled both the dominant and nondominant arms together to compare between baseball players and nonthrowing controls. Therefore, it is difficult to compare the results of their study with those of our current study. Future studies are needed to examine acromiohumeral distance prospectively to determine if participation in overhead sports causes changes overtime.

The scapula has gained substantial attention in the past decade in regard to overuse shoulder injuries. ${ }^{1,9,22-25}$ Several studies have demonstrated alterations in scapular position or motion in injured and healthy overhead athletes. ${ }^{8,9,19,26}$ These studies have commonly observed 
decreased amounts of scapular upward rotation, $8,9,19,27$ which is thought to decrease the subacromial space by approximating the acromion with the humerus. However, this relationship has yet to be determined in healthy college baseball players.

The results of the current study did not demonstrate a significant correlation between acromiohumeral distance and scapular upward rotation at either $0^{\circ}$ or $90^{\circ}$ of glenohumeral abduction. This finding suggests that there is no relationship between the amount of scapular upward rotation present and the acromiohumeral distance in healthy baseball players. Clinically, this is an important finding due to the strong assumption that any decreases in scapular upward rotation place the acromion closer to the humerus. This is then thought to cause shoulder injuries such as impingement and rotator-cuff tears in overheadthrowing athletes due to increased compression force on the soft-tissue structures in the subacromial space. . $^{8,9,19}$ One study has examined subacromial clearance through the use of contact forces in cadaver shoulders and the effect of scapular motion on subacromial clearance. ${ }^{28}$ Those researchers found that the subacromial clearance was decreased as scapular upward rotation was increased. This finding goes against previous theories, which describe increased clearance with increased scapular upward rotation. ${ }^{9,24,29}$ Our study does not agree with the current in vivo theory or the findings of the cadaver study; however, Karduna et $\mathrm{al}^{28}$ examined this in cadavers with simulated muscle activation of the rotator cuff and deltoid. The effect of muscle contraction or at minimum baseline muscle tone may play a large part in this relationship. Muscle contractions have been shown to cause large translations of the humeral head during motion, potentially even leading to shoulder dislocation. ${ }^{30}$ These strong muscle contractions that are often observed during overhead throwing may be affecting the acromiohumeral distance. However, during testing only minimal muscle contractions are required to support the arm and therefore may not adequately represent the true relationship during functional movements such as throwing. Another possible explanation for the lack of a relationship between scapular upward rotation and acromiohumeral distance may be glenoid inclination. It has been shown that individuals with a larger glenoid inclination angle have a higher risk of rotator-cuff tears. ${ }^{31}$ Overhead athletes with a larger glenoid inclination angle may have less acromiohumeral distance due to the orientation of their glenoid and not due to changes in scapular upward rotation. Unfortunately, this was not measured in the current study because of the need for radiographs, but future studies should examine this in healthy overhead athletes to provide a more detailed analysis of the effect on acromiohumeral distance and potentially rotator-cuff injury. Due to the results of our current study and others, it seems likely that the acromiohumeral distance is multifactorial in nature, and scapular upward rotation alone does not contribute to changes in healthy individuals. Every variable discussed may play a role in changes in acromiohumeral distance, and in the future should all be examined to determine the relative contributions of each. This will help clinicians better weigh each alteration to concentrate their efforts in preventing and managing overuse shoulder injuries in overhead-throwing athletes.

\section{Limitations}

There are some limitations to this study that should be acknowledged. First, both pitchers and position players were combined and analyzed together. It has been demonstrated in the literature that pitchers subject their shoulders to additional stress due to the high number of repetitions in each game. However, in a recent study that was conducted in our laboratory measuring scapular upward rotation, no differences were observed between pitchers and position players. ${ }^{32} \mathrm{Next}$, only 1 of the possible 5 degrees of freedom was measured for the scapula. This is a limitation due to the potential injury risk of alterations in the other motions of the scapula. Posterior tilting of the scapula has been thought to correlate with acromiohumeral distance, as well, and may be another factor that needs to be considered in the future. However, to our knowledge scapular upward rotation is the only valid and reliable measurement that can be observed clinically. ${ }^{20,33-35}$ Currently, a 3-dimensional electromagnetic tracking system can be used to measure all five degrees of scapular motion. However, this technique does not translate well to a clinician and still has some question as to its validity and reliability due to skin motion between the sensor and the scapula. ${ }^{36,37}$

\section{Conclusion}

Healthy college baseball players do not present with acromiohumeral-distance differences when comparing the dominant arm with the nondominant arm. These results indicate that the acromiohumeral distance is not adapting in the dominant arm in healthy throwing athletes; this may suggest that changes that are typically seen in an injured population may be occurring due to the injury. However, prospective studies are needed to further monitor overhead athletes over time to determine if the acromiohumeral distance is adaptively changing. A relationship was not identified between acromiohumeral distance and scapular upward rotation. This suggests that scapular upward rotation may not be the only contributing factor to acromiohumeral distance. Other factors such as scapular posterior tilting, the level of muscle contraction, and glenoid inclination may all play roles in the acromiohumeral distance and should be examined in the future to determine the relative contribution of each.

\section{References}

1. Su KPE, Johnson MP, Gracely EJ, Karduna AR. Scapular rotation in swimmers with and without impingement syndrome: practice effects. Med Sci Sports Exerc. 2004;36:1117-1123. PubMed doi:10.1249/01. MSS.0000131955.55786.1A 
2. Tyler TF, Nicholas SJ, Roy T, Gleim GW. Quantification of posterior capsule tightness and motion loss in patients with shoulder impingement. Am J Sports Med. 2000;28:668-673. PubMed

3. Myers JB, Laudner KG, Pasquale MR, Bradley JP, Lephart SM. Glenohumeral range of motion deficits and posterior shoulder tightness in throwers with pathologic internal impingement. Am J Sports Med. 2006;34(3):385-391. PubMed doi:10.1177/0363546505281804

4. Jobe CW, Pink MM, Jobe FW. Anterior shoulder instability, impingement, and rotator cuff tear: theories and concepts. In: Jobe FW, ed. Operative Techniques in Upper Extremity Sports Injuries. St Louis, MO: Mosby; 1996:164-176.

5. Jobe FW, Kvitne RS, Giangarra CE. Shoulder pain in the overhand or throwing athlete: the relationship of anterior instability and rotator cuff impingement. Orthop Rev. 1989;18(9):963-975. PubMed

6. Cavallo RJ, Speer KP. Shoulder instability and impingement in throwing athletes. Med Sci Sports Exerc. 1998;30:S18-S25. PubMed

7. Neer CS. Anterior acromioplasty for the chronic impingement syndrome in the shoulder: a preliminary report. $J$ Bone Joint Surg Am. 1972;54:41-50. PubMed

8. Lukasiewicz AC, McClure PW, Michener L, Pratt N, Sennett B. Comparison of 3-dimensional scapular position and orientation between subjects with and without shoulder impingement. J Orthop Sports Phys Ther. 1999;29:574-583. PubMed

9. Laudner KG, Myers JB, Pasquale MR, Bradley JP, Lephart SM. Scapular dysfunction in throwers with pathologic internal impingement. J Orthop Sports Phys Ther. 2006;36(7):485-494. PubMed

10. Ludewig PM, Cook TM. Alterations in shoulder kinematics and associated muscle activity in people with symptoms of shoulder impingement. Phys Ther. 2000;80:276-291. PubMed

11. McClure PW, Michener LA, Karduna AR. Shoulder function and 3-dimensional scapular kinematics in people with and without shoulder impingement syndrome. Phys Ther. 2006;86(8):1075-1090. PubMed

12. Thomas SJ, Swanik CB, Swanik K, Kelly JD. Change in glenohumeral rotation and scapular position after a Division I collegiate baseball season. J Sport Rehabil. 2013;22(2):115-121.

13. Thomas SJ, Swanik KA, Swanik CB, Huxel KC, Kelly JD. Internal rotation and scapular position changes following competitive high school baseball. J Sport Rehabil. 2010;19(2):125-135. PubMed

14. Thomas SJ, Swanik KA, Swanik CB, Kelly JD. Internal rotation and scapular position differences: a comparison of collegiate and high school baseball players. J Athl Train. 2010;45(1):44-50. PubMed doi:10.4085/10626050-45.1.44

15. Michener LA, McClure PW, Karduna AR. Anatomical and biomechanical mechanisms of subacromial impingement syndrome. Clin Biomech (Bristol, Avon). 2003;18(5):369-379. PubMed doi:10.1016/S02680033(03)00047-0
16. Desmeules F, Minville L, Riederer B, Cote CH, Fremont P. Acromio-humeral distance variation measured by ultrasonography and its association with the outcome of rehabilitation for shoulder impingement syndrome. Clin J Sport Med. 2004;14(4):197-205. PubMed doi:10.1097/00042752-200407000-00002

17. Neer CS. Impingement lesions. Clin Orthop Relat Res. 1983; (173):70-77. PubMed

18. Wang HK, Lin JJ, Pan SL, Wang TG. Sonographic evaluations in elite college baseball athletes. Scand J Med Sci Sports. 2005;15(1):29-35. PubMed doi:10.1111/j.16000838.2004.00408.x

19. Thomas SJ, Swanik KA, Swanik C, Huxel KC. Glenohumeral rotation and scapular position adaptations after a single high school female sports season. J Athl Train. 2009;44(3):230-237. PubMed doi:10.4085/1062-605044.3.230

20. Johnson MP, McClure PW, Karduna AR. New method to assess scapular upward rotation in subjects with shoulder pathology. J Orthop Sports Phys Ther. 2001;31:81-89. PubMed

21. Hebert LJ, Moffet H, Dufour M, Moisan C. Acromiohumeral distance in a seated position in persons with impingement syndrome. J Magn Reson Imaging. 2003;18(1):72-79. PubMed doi:10.1002/jmri.10327

22. Kibler BW. Management of the scapula in glenohumeral instability. Tech Shoulder Elbow Surg. 2003;4:89-98. doi:10.1097/00132589-200309000-00001

23. Kibler WB, McMullen J. Scapular dyskinesis and its relation to shoulder pain. J Am Acad Orthop Surg. 2003;11:142-151. PubMed

24. Burkhart SS, Morgan CD, Kibler BW. The disabled throwing shoulder: spectrum of pathology, part III: the SICK scapula, scapular dyskinesis, the kinetic chain, and rehabilitation. Arthroscopy. 2003;19:641-661. PubMed doi:10.1016/S0749-8063(03)00389-X

25. Myers JB, Laudner KG, Pasquale MR, Bradley JP, Lephart SM. Scapular position and orientation in throwing athletes. Am J Sports Med. 2005;33:263-271. PubMed doi:10.1177/0363546504268138

26. Warner JJP, Micheli LJ, Arslenian LE, Kennedy J, Kennedy R. Scapulothoracic motion in normal shoulders and shoulders with glenohumeral instability and impingement syndrome: a study using Moiré topographic analysis. Clin Orthop Relat Res. 1992; (285):191-199. PubMed

27. Laudner KG, Stanek JM, Meister K. Differences in scapular upward rotation between baseball pitchers and position players. Am J Sports Med. 2007;35(12):2091-2095. PubMed doi:10.1177/0363546507305098

28. Karduna AR, Kerner PJ, Lazarus MD. Contact forces in the subacromial space: effects of scapular orientation. J Shoulder Elbow Surg. 2005;14(4):393-399. PubMed doi:10.1016/j.jse.2004.09.001

29. Kibler WB. Scapular involvement in impingement: signs and symptoms. Instr Course Lect. 2006;55:35-43. PubMed

30. McMahon PJ, Lee TQ. Muscles may contribute to shoulder dislocation and stability. Clin Orthop Relat Res. 2002; 
(403, Suppl)S18-S25. PubMed doi:10.1097/00003086200210001-00003

31. Hughes RE, Bryant CR, Hall JM, et al. Glenoid inclination is associated with full-thickness rotator cuff tears. Clin Orthop Relat Res. 2003; (407):86-91. PubMed doi:10.1097/00003086-200302000-00016

32. Thomas SJ, Swanik KA, Swanik C. Internal rotation and total motion differences: a comparison of baseball pitchers and position players. J Athl Train. 2008;43(3):s-21.

33. Kibler BW. Role of the scapula in the overhead throwing motion. Contemp Orthop. 1991;22:525-532.

34. Kibler WB. The role of the scapula in athletic shoulder function. Am J Sports Med. 1998;26:325-337. PubMed
35. Borsa PA, Timmons MK, Sauers EL. Scapular-positioning patterns during humeral elevation in unimpaired shoulders. J Athl Train. 2003;38:12-17. PubMed

36. McClure PW, Michener LA, Sennett B, Karduna AR. Direct 3-dimensional measurement of scapular kinematics during dynamic movements in vivo. J Shoulder Elbow Surg. 2001;10:269-277. PubMed doi:10.1067/ mse.2001.112954

37. Ludewig PM, Phadke V, Braman JP, Hassett DR, Cieminski CJ, LaPrade RF. Motion of the shoulder complex during multiplanar humeral elevation. J Bone Joint Surg Am. 2009;91(2):378-389. PubMed doi:10.2106/ JBJS.G.01483 